\title{
TWO INFINITE-FROUDE-NUMBER CUSPED FREE-SURFACE FLOWS DUE TO A SUBMERGED LINE SOURCE OR SINK
}

\author{
I. L. COLLINGS ${ }^{1}$
}

(Received 4 July 1985; revised 25 September 1985)

\begin{abstract}
Solutions are found to two cusp-like free-surface flow problems involving the steady motion of an ideal fluid under the infinite-Froude-number approximation The flow in each case is due to a submerged line source or sink, in the presence of a solid horizontal base.
\end{abstract}

\section{Introduction}

Tuck and Vanden Broeck [4] and, more recently, Hocking [2] and Yih [5], have considered a series of two-dimensional free-surface flow problems having in common a submerged line source or sink beneath a cusped free surface. They have assumed the flow to be that of an ideal fluid moving irrotationally under gravity, and have shown that steady-state solutions having a cusped, monotonic free-surface horizontal at infinity exist at a unique Froude number, or over a limited range. In particular, [4] used conformal mapping techniques to obtain a solution to the problem outlined above, when the line source or sink lies at a finite depth in an infinitely deep fluid. They showed that a solution exists for a unique Froude number, namely 1.776 .

\footnotetext{
${ }^{1}$ Division of Computing \& Mathematics, Deakin University, Victoria 3217, Australia.

(c) Copyright Australian Mathematical Society 1986, Serial-fee code 0334-2700/86
} 


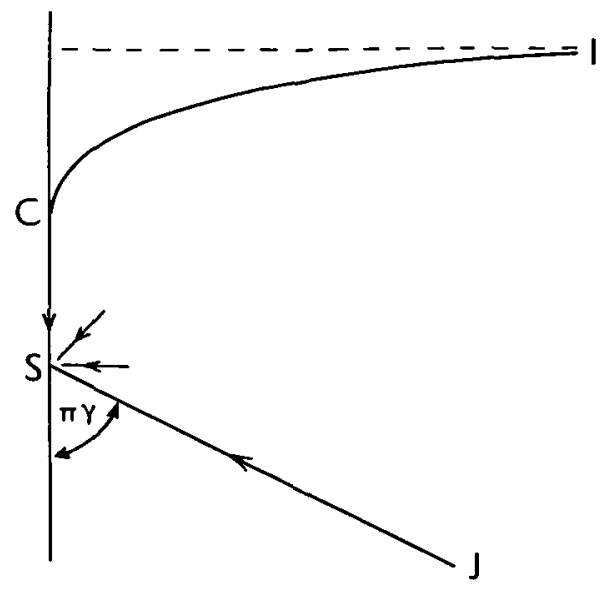

Figure 1 . The physical $z$ plane, with the sink $S$ at the mountain peak.

Hocking [2] adopted the numerical technique used in [4] to examine the problem when the source or sink lies on the top of an infinite triangular mountain of semi-apex angle $\pi \gamma$, the geometry of which is shown in Figure 1. The problem considered by Tuck and Vanden Broeck [4] is that obtained when $\gamma=0$. Hocking solved for $\gamma$ in the range $0 \leqslant \gamma \leqslant \frac{1}{2}$, and showed that for any given $\gamma$ a solution exists, but for a single Froude number only. Yih [5], in a study of the critical Froude number associated with selective withdrawal into a sink, adopted and modified the formula used by Richardson [3] in which the geometry of the bottom and the exact location of the sink are determined a posteriori. He showed, inter alia, that when Richardson's formula is used, the condition that the bottom be horizontal, with the sink on the bottom, can only be satisfied if the Froude number is infinite. This problem is considered again here in detail in Section 2, using an infinite-Froude-number formulation. As mentioned above, the problem with the sink on a horizontal plane has been solved by Hocking for finite Froude number. Yih also showed that, if the constraint of a flat bottom is relaxed, then many solutions are possible, whether the sink is on the bottom or not. In addition, Hocking solved the problem when the line source or sink lies above a horizontal plane. (The case where the source or sink lies on the plane is his mountain-peak geometry with $\gamma=\frac{1}{2}$.) He showed that in this case solutions exist over a range of Froude number, namely $1 \leqslant F \leqslant 1.44$.

This note was prompted by the papers of Tuck and Vanden Broeck [4] and Hocking [2], and addresses the infinite-Froude-number flows associated with two simple geometries chosen for their analytic convenience. We consider a line sink on a horizontal plane and as a separate problem a line sink above a horizontal plane, both under the infinite-Froude-number approximation. The work in both 
[2] and [4] suggests that solutions will not exist as $F \rightarrow \infty$, although the recent paper by Yih [5] shows that this is not the case, at least when the sink is on the plane. This is confirmed in the present study and indicates that the results obtained by [2] and [4] are limited to finite values of $F$. We find in both problems that a single solution exists in terms of the geometry in the physical $z$ plane. This is essentially the same result as found in [2] and [4], since the reference length in the definition of their Froude numbers is based on either the depth of the sink or the depth of the horizontal base below the (horizontal) free surface at infinity.

\section{A sink on a horizontal plane}

In this and the following sections, reference to a sink means either a horizontal line sink or line source. In this section, we consider a sink on a horizontal plane, with a cusped free surface directly above the sink. The free-surface condition of constant pressure is replaced by a condition of constant speed under the infiniteFroude-number approximation, and the usual hodograph techniques are applicable (see, for example, [1]).

We let $z=x+i y$ represent the physical $z$ plane and place the origin at the cusp. In the usual notation $w=\varphi+i \psi$ is the complex velocity potential and the hodograph plane is defined by

$$
\zeta=d z / d w=e^{\imath \theta} / q,
$$

where $q$ and $\theta$ give the speed and direction of the flow respectively. Note that $|d z / d w|=1$ is the non-linear boundary condition on the free surface. When gravity is not present, the use of the hodograph plane linearises the problem since the free surface maps onto a quarter arc of the circle $u^{2}+v^{2}=1$, and the solution may be obtained by complex variable methods. The solution domains in the $z, w$ and $\zeta$ planes are shown in Figure 2. The variables are non-dimensional, where we have non-dimensionalised the coordinates by $m / q_{0}$, the complex velocity potential by $m$, and the complex velocity by $q_{0}$. Here $q_{0}$ is the constant speed on the free surface, and $m$ is the volumetric flux per unit thickness, which is equal to $q_{0} h$, where $h$ is the fluid depth at infinity. It is not hard to show (mapping via a parametric half-plane) that

$$
e^{\pi w}=-4 \zeta^{2} /\left(1-\zeta^{2}\right)^{2}
$$

which incidentally is equivalent to equation (2.1) of Tuck and Vanden Broeck [4], with their $t$ replaced by $-\zeta^{2}$. From (2.1) and (2.2), we have at once

$$
z=\int_{0}^{w} \zeta d w
$$


where

$$
\zeta=\left[1-2 e^{-\pi w}+2\left(e^{-2 \pi w}-e^{-\pi w}\right)^{1 / 2}\right]^{1 / 2}
$$

Note from (2.1), using (2.4), that $d z \sim d w$ as $\varphi \rightarrow+\infty, \psi=0$, and so the jump in $\psi$ from 0 to -1 at $I, J$ in the $w$ plane corresponds to a jump in $y$ of -1 at usptream infinity in the $z$ plane. This result can of course be obtained directly from non-dimensional flux considerations, given that the speed on the free surface is constant and equal to 1 , and that the flow tends towards a uniform stream at large distances from the sink.

We now proceed to determine the nature of the free surface, which is given by $0 \leqslant \varphi<\infty, \psi=0$ in the $w$ plane. From (2.3) and (2.4), a point $z\left(\varphi^{*}\right)$ on the free
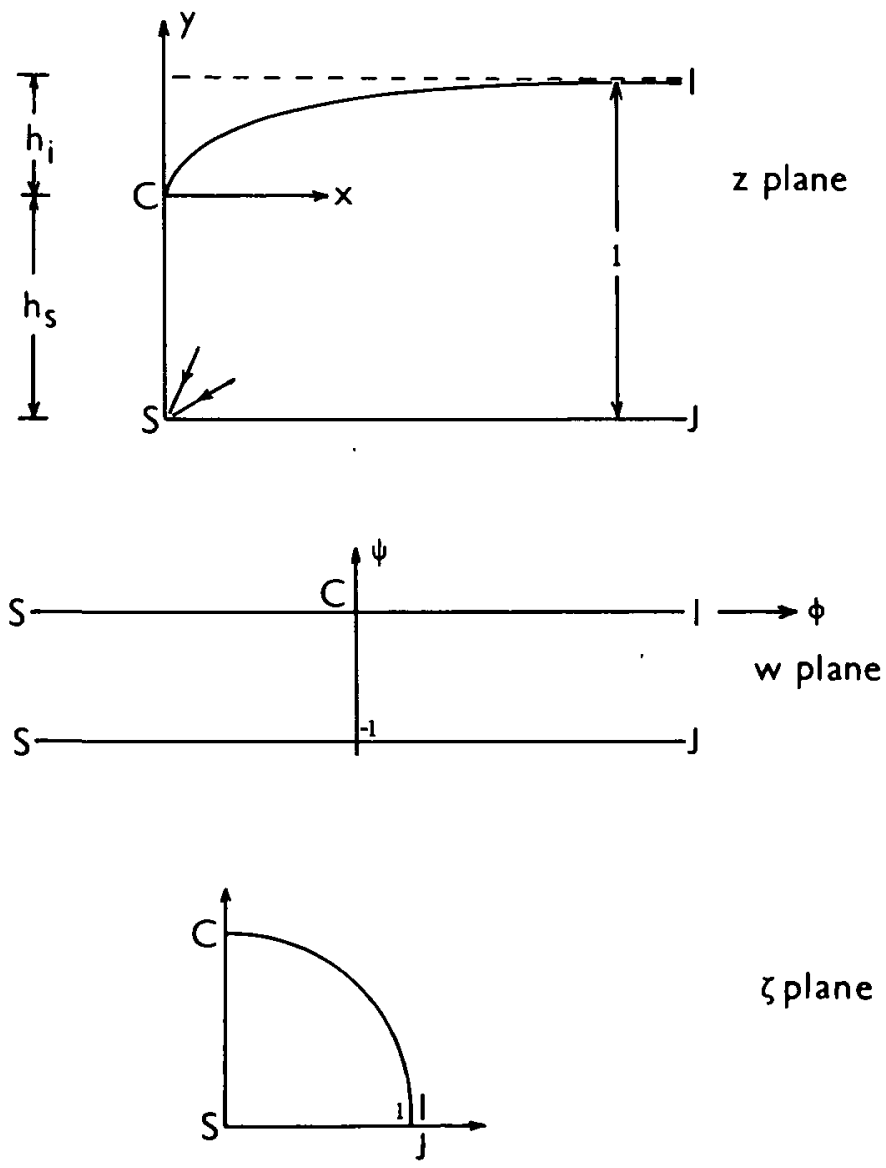

$\zeta$ plane

Figure 2. Flow regions in the non-dimensional $z, w$ and $\zeta$ planes, for a sink on a horizontal plane. 
surface is

$$
z\left(\varphi^{*}\right)=\int_{0}^{\varphi^{*}}\left[1-2 e^{-\pi \varphi}+2 i\left(e^{-\pi \varphi}-e^{-2 \pi \varphi}\right)^{1 / 2}\right]^{1 / 2} d \varphi
$$

since $0 \leqslant \varphi^{*}<\infty$. For small $\varphi$, the integrand in (2.5) may be approximated by $i+(\pi \varphi)^{1 / 2}$, and so

$$
z \sim \int_{0}^{\varphi^{*}}\left[i+(\pi \varphi)^{1 / 2}\right] d \varphi
$$

for small $\varphi$, which gives

$$
x \sim 2 \pi^{1 / 2}\left(\varphi^{*}\right)^{3 / 2} / 3, \quad y \sim \varphi^{*},
$$

or

$$
y=\pi^{-1 / 3}(3 x / 2)^{2 / 3},
$$

a cusped free surface near the origin, as required.

A similar analysis may be carried out for large $\varphi$, from which it may be shown that

$$
x \sim \varphi^{*}, \quad y \sim \frac{2}{\pi}\left(1-e^{-\pi \varphi^{*} / 2}\right) \rightarrow \frac{2}{\pi} \quad \text { as } \varphi^{*} \rightarrow \infty .
$$

The distance $h$, between the origin and the level of the free surface at upstream infinity (see Figure 2 ) is therefore $2 / \pi$. This result has already been obtained by Yih [5] in his finite-Froude-number solution, by letting $F \rightarrow \infty$. The results in (2.6) and (2.8) have been verified by the numerical integration of (2.5) using Simpson's rule.

The distance between the cusp and the source, again using (2.3) and (2.4), is

$$
-i h_{s}=\int_{0}^{-\infty}\left[1-2 e^{-\pi \varphi}+2\left(e^{-2 \pi \varphi}-e^{-\pi \varphi}\right)^{1 / 2}\right]^{1 / 2} d \varphi=-0.36338 i
$$

the integration being performed using an IMSL numerical-integration package in double-precision arithmetic. The result is correct ot 5 decimal places. Hence $h_{s}=0.36338$ and so $h_{i}+h_{s}=1$, in agreement with our non-dimensional flux requirement at infinity.

The depth of the cusp is $2 / \pi$ times, or $63.66 \%$ of, the depth of the source beneath the level of the free surface at infinity. This compares with $85.44 \%$ obtained by Hocking [2] when gravity is included.

In conclusion, a solution to the specified problem exists for a unique geometrical configuration. In [2], a solution under gravity was found for a unique finite Froude number, with a length scale based on the depth of the source below the level of the free surface at infinity. The results are qualitatively the same. The inverse approach of Yih [5] using Richardson's [3] formula gives a solution, but 
only for infinite $F$. It would appear that the more-direct methods of Tuck and Vanden Broeck [4] and Hocking [2] should lead to solutions giving lower values of $F$ for any given linear bottom geometry. We now turn our attention to the flow when the sink is above a horizontal plane.

\section{A sink above a horizontal plane}

We use standard conformal mapping techniques as in Section 2, except that the hodograph plane is no longer appropriate, due to the discontinuity in the direction of the flow at the stagnation point $B$ on the horizontal plane directly below the sink. This difficulty is overcome if we use a logarithmic hodograph
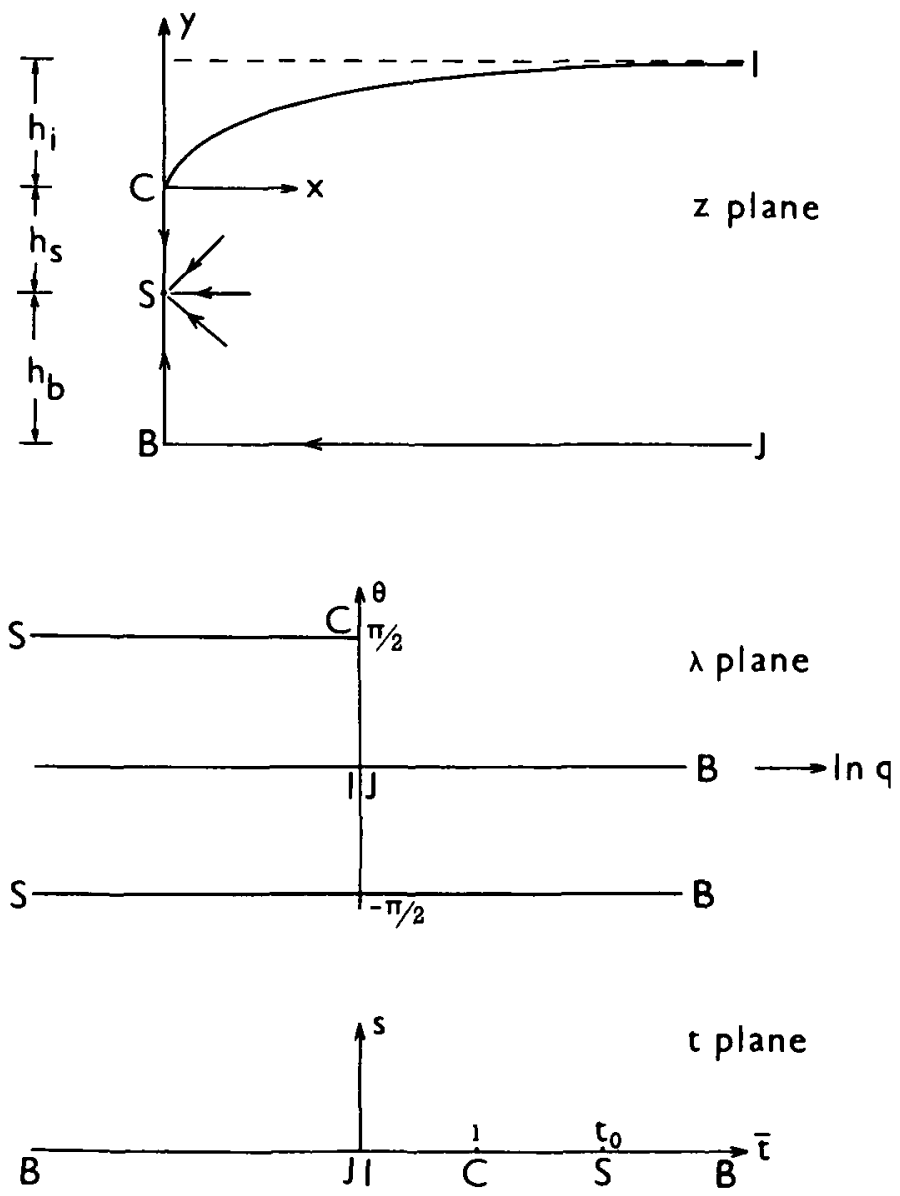

Figure 3. Flow regions in the non-dimensional $z$ and $\lambda$ planes and the parametric $t$ plane, for a sink above a horizontal plane. 
plane defined by

$$
\lambda=\ln \left(\frac{d z}{d w}\right)=\ln q^{-1}+i \theta .
$$

The $w$ plane is that shown in Figure 2, with a point $B$ inserted on the line $\psi=-1$ between $S$ and $J$. We map the solution domains in the $\lambda$ and $w$ planes onto the upper half of a parametric $t$ plane. The $z, \lambda$ and $t$ planes are shown in Figure 3.

The conformal mapping between the $w$ and $t$ planes is given by

$$
w=\frac{1}{\pi} \ln \frac{t-t_{0}}{t\left(t_{0}-1\right)}-i, \quad t_{0}>1
$$

and so

$$
d w / d t=t_{0} /\left[\pi t\left(t-t_{0}\right)\right] .
$$

The mapping between the $\lambda$ and $t$ planes effected by the Schwarz-Christoffel transformation is given by the integral

$$
\lambda=A \int \frac{t^{1 / 2} d t}{(t-1)^{1 / 2}\left(t-t_{0}\right)}+B
$$

where $A, B$ are (complex) constants to be determined. If we concern ourselves initially (as in Section 2) with the free surface where $1 \geqslant \bar{t} \geqslant 0$, we write $(t-1)^{1 / 2}$ in the integrand of $(3.3)$ as $i(1-t)^{1 / 2}$, to obtain

$$
\lambda=\frac{\pi i}{4}-i A\left[\arcsin (2 t-1)+\left(\frac{t_{0}}{t_{0}-1}\right)^{1 / 2} \arcsin \frac{t_{0}-2 t_{0} t+t}{t_{0}-t}\right],
$$

where

$$
A=\frac{1}{2\left\{\left(t_{0} /\left(t_{0}-1\right)\right)^{1 / 2}-1\right\}} .
$$

The constants $A, B$ in (3.3) have been determined by requiring that $\lambda(0)=0$, $\lambda(1)=\pi i / 2$.

From (3.1), a point $z\left(t^{*}\right)$ on the free surface is given by

$$
z\left(t^{*}\right)=\int_{1}^{t^{*}} e^{\lambda} \frac{d w}{d t} d t
$$

where $t^{*}$ is real and such that $1 \geqslant t^{*} \geqslant 0$ and $\lambda$ and $d w / d t$ are given by (3.4) and (3.2) respectively. Integrals along the real axis in the complex $t$ plane should be written with respect to $\bar{t}$. We shall drop the "bar" notation without confusion.

For

$$
t \leq 1, \quad \lambda \sim \frac{\pi i}{2}-\frac{2 i A(1-t)^{1 / 2}}{t_{0}-1} \text { and } \frac{d w}{d t} \sim \frac{-t_{0}}{\pi\left(t_{0}-1\right)}
$$


Hence if $t^{*} \leq 1$, that is, near the point $C$ in the physical $z$ plane,

$$
z\left(t^{*}\right) \sim \frac{t_{0}}{\pi\left(t_{0}-1\right)}\left[\frac{4 A\left(1-t^{*}\right)^{3 / 2}}{3\left(t_{0}-1\right)}+i\left(1-t^{*}\right)\right]
$$

which gives

$$
x \sim \frac{4 A t_{0}\left(1-t^{*}\right)^{3 / 2}}{3 \pi\left(t_{0}-1\right)^{2}}, \quad y \sim \frac{t_{0}\left(1-t^{*}\right)}{\pi\left(t_{0}-1\right)}
$$

and which is equivalent to

$$
y=(3 /(4 A))^{2 / 3}\left[t_{0}\left(t_{0}-1\right) / \pi\right]^{1 / 3} x^{2 / 3},
$$

a cusped free surface as required. An asymptotic expression for the free surface at upstream infinity is not readily obtainable; however the integrand in (3.6) may easily be broken into real and imaginary parts and was integrated for various $t_{0}>1$ using a NAG numerical-integration package. The numerical results confirmed (3.7) and showed the free surface to be asymptotic in $y$ as $t^{*} \rightarrow 0$, as required. We now turn our attention to the distance $h_{s}$ of the sink below the origin and the distance $h_{b}$ between the sink and the horizontal plane.

As (3.4) is valid for $0 \leqslant t \leqslant 1$, we take its analytic continuation outside this interval to obtain

$$
\lambda=\frac{\pi i}{2}-A\left[-\ln \left[\Delta_{1}+\left(\Delta_{1}^{2}-1\right)^{1 / 2}\right]+\left(\frac{t_{0}}{t_{0}-1}\right)^{1 / 2} \ln \left[-\Delta_{1}+\left(\Delta_{1}^{2}-1\right)^{1 / 2}\right]\right]
$$

for $1 \leqslant t<t_{0}$, and

$$
\lambda=\frac{-\pi i}{2}-A\left[-\ln \left[\Delta_{1}+\left(\Delta_{1}^{2}-1\right)^{1 / 2}\right]+\left(\frac{t_{0}}{t_{0}-1}\right)^{1 / 2} \ln \left[\Delta_{2}+\left(\Delta_{2}^{2}-1\right)^{1 / 2}\right]\right]
$$

for $t>t_{0}$, where

$$
\Delta_{1}=2 t-1, \quad \Delta_{2}=1-2 t-\frac{2 t(t-1)}{t_{0}-t} .
$$

From (3.1) we obtain

$$
-i h_{s}\left(t_{0}\right)=\int_{1}^{t_{0}} e^{\lambda} \frac{d w}{d t} d t
$$


where $\lambda$ is given by (3.9), and

$$
-i h_{b}\left(t_{0}\right)=\int_{t_{0}}^{\infty} e^{\lambda} \frac{d w}{d t} d t
$$

where $\lambda$ is given by (3.10). From (3.6) we have

$$
h_{\imath}\left(t_{0}\right)=\operatorname{Im} \int_{1}^{0} e^{\lambda} \frac{d w}{d t} d t
$$

where $\lambda$ is given by (3.4). The only constraint now to be met is the non-dimensional flux condition $h_{i}+h_{s}+h_{b}=1$, and it was thought that this condition would be satisfied for all $t_{0}>1$ and so an infinite number of geometries satisfy the problem. However, the integrand in (3.13) behaves like $t^{A-2}$ for large $t$ and hence will be convergent provided $2-A>1$, that is, $t_{0}<9 / 5$. Hocking [2] showed when gravity was included in the problem that solutions existed for a range of Froude number and it was thought that solutions might exist for a range of $t_{0}$ in the interval $1<t_{0}<1.8$. The integrals (3.12), (3.13) and (3.14) were evaluated for $t_{0}$ in this interval and the sum $I=h_{t}+h_{s}+h_{b}$ is shown plotted against $t_{0}$ in Figure 4. Due to flux considerations, the only acceptable value for $I$ is 1. As shown in Figure 4, the minimum value of $I$ is 1.00000 (correct to 5 decimal places) which occurs when $t_{0}=1.332$.

As in Section 2 we obtain a unique geometry. The values of $h_{t}, h_{s}$ and $h_{b}$ when $t_{0}=1.332$ are $0.26326,0.16932$ and 0.56742 respectively, giving a cusp-to-source depth ratio below the level of the uniform stream at infinity of $60.857 \%$. Hocking obtained ratios between $70 \%$ and $80 \%$ for his range of Froude number. A plot of the free surface is shown in Figure 5.

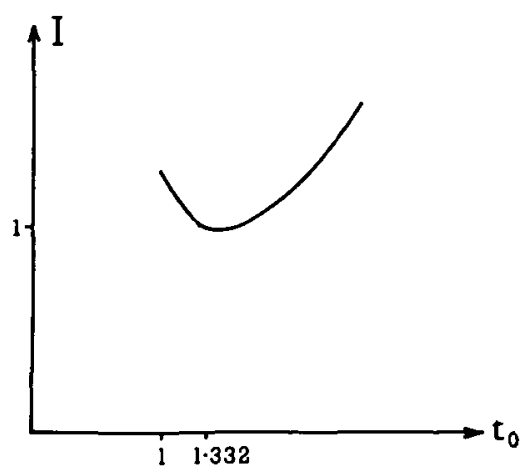

Figure 4. Plot of $I=h_{t}+h_{s}+h_{b}$ against $t_{0}$. 


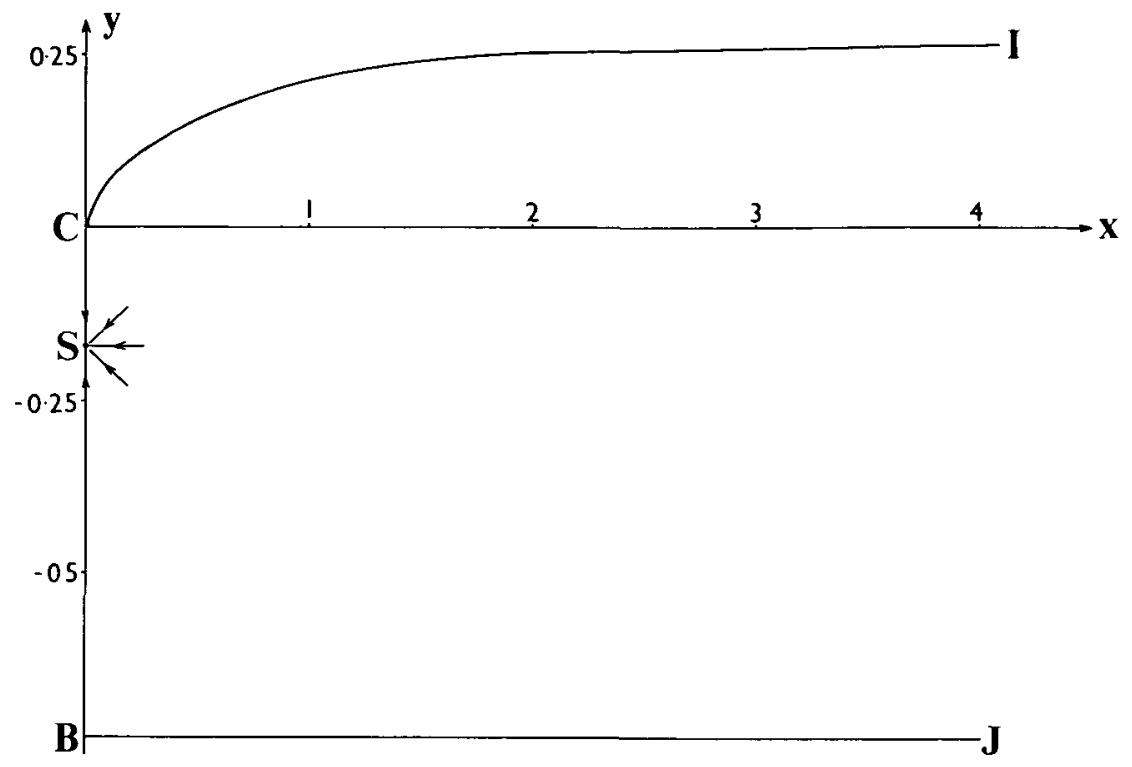

Figure 5. Plot of the free surface, for a sink above a horizontal plane.

It is worth noting that the problem solved in Section 2 cannot be obtained as a limiting case of the problem solved here. To let the source $S$ approach the base $B$ we want $h_{b} \rightarrow 0$ and this requires $t_{0} \rightarrow \infty$ in Figure 3. As described above, the integral (3.13) diverges when $t_{0}>1.8$. Furthermore, the logarithmic hodograph plane for the problem in Section 2 is a semi-infinite rectangular strip, which is not obtainable as a special or limiting case of the $\lambda$ plane shown in Figure 3.

\section{Conclusion}

This note was prompted by the papers of Tuck and Vanden Broeck [4] and Hocking [2] who considered cusp-like free-surface flows under gravity due to a submerged line source or sink. Two flows studied in [2] have been considered here under the infinite-Froude-number approximation and it is shown that solutions do exist, a result which appears at first to be contrary to the conclusions in both [2] and [4]. It should be noted, however, that the work of these authors is restricted to finite Froude number and that their results are applicable for finite $F$ only.

The case where the source or sink is above the horizontal bed is interesting, since Hocking [2] obtained solutions for a range of $F$ and not for a unique $F$ as in other problems he considered. Given that the infinite- $F$ problem has a solution 
for a single geometry only, it is curious that the solution to the finite- $F$ problem has a range of geometries. It is not clear why this should be the case once a flux constraint based on the strength of the source or sink is included.

Finally, the results here cover the infinite- $F$ approximation to cusped, steadystate free-surface problems due to a submerged line source of sink in the presence of a solid horizontal bed. Solutions of the type described here will not exist for a sink in a fluid of infinite depth, since the constant velocity on the free surface would then be zero, assuming of course that the strength of the sink is finite.

\section{Acknowledgements}

The author wishes to thank the referees and editor for their helpful comments and criticism.

\section{References}

[1] G. Birkhoff and E. H. Zarantonello, Jets, wakes and cavities (Academic Press, New York, 1957).

[2] G. C. Hocking, “Cusp-like free-surface flows due to a submerged source or sink in the presence of a flat or sloping bottom", J. Austral. Math. Soc. Ser. B. 26 (1985), 470-486.

[3] A. R. Richardson, "Stationary waves in water", Phil. Mag. (6) 40 (1920), 97-110.

[4] E. O. Tuck and J. M. Vanden Broeck, "A cusp-like free-surface flow due to a submerged source or sink", J. Austral. Math. Soc. Ser. B. 25 (1984), 443-450.

[5] C. S. Yih, "Effect of geometry on selective withdrawal" (to appear in J. Fluid Mech.). 